\title{
STRAIN BALANCED QUANTUM POSTS FOR INTERMEDIATE BAND SOLAR CELLS
}

\author{
D. Alonso-Álvarez* ${ }^{*}$, B. Alén ${ }^{1}$, J. M. Ripalda ${ }^{1}$, A. G. Taboada ${ }^{1}$, J. M. Llorens ${ }^{1}$, Y. González ${ }^{1}$, L. González ${ }^{1}$, F. Briones ${ }^{1}$, E. \\ Antolín $^{2}$, I. Ramiro ${ }^{2}$, A. Martí ${ }^{2}$, A. Luque ${ }^{2}$, M. A. Roldán ${ }^{3}$, J. Hernandez-Saz ${ }^{3}$, M. Herrera ${ }^{3}$ and S.I.Molina ${ }^{3}$ \\ ${ }^{1}$ Instituto de Microelectrónica de Madrid, CNM (CSIC), c/lsaac Newton 8, PTM, Tres Cantos, 28760 Madrid, Spain. \\ ${ }^{2}$ Instituto de Energía Solar, IES-UPM.Ciudad Universitaria,28040, Madrid, Spain. \\ ${ }^{3}$ Departamento de Ciencia de los Materiales e Ing. Metalúrgica y Q. I. Universidad de Cádiz, Campus Universitario de \\ Puerto Real, 11510 Puerto Real, Cádiz, Spain.
}

\begin{abstract}
In this work we present strain balanced InAs quantum post of exceptional length in the context of photovoltaics. We discuss the general properties of these nanostructures and their impact in the practical implementation of an intermediate band solar cell. We have studied the photocurrent generated by strain balanced quantum posts embedded in a GaAs single crystal, and compared our results with quantum dot based devices. The incorporation of phosphorous in the matrix to partially compensate the accumulated stress enables a significant increase of the quantum post maximum length. The relative importance of tunneling and thermal escape processes is found to depend strongly on the geometry of the nanostructures.
\end{abstract}

\section{INTRODUCTION}

Over the last decades semiconductor nanostructures have been suggested as a means to increase the efficiency of solar cells. Strain balanced multi-quantum-wells (SBMQW) have been successfully implemented as the bottom GaAs cell of a current matched tandem configuration to tailor the absorption edge and increase the overall efficiency.[1] In the case of quantum dots (QDs), there are different approaches that may lead to a better conversion of the sunlight into electricity, being one of them the intermediate band solar cell concept (IBSC).[2, 3] Recent detailed balance calculations, based in more realistic assumptions than previous results and including an intermediate band with multiple levels thermally interconnected, seem to indicate that even InAs/GaAs QDs, with bandgaps different from the optimum ones, can exhibit higher efficiency than a single gap cell at 1000 suns.[4] Yet, despite the existing effort to model QDs correctly in the context of IBSC theory, the experimental achievements are still far from the predicted values.

One of the main disadvantages of QDs is their small density and, hence, their reduced contribution to the total photocurrent. Several tens of QDs layers are necessary to absorb an appreciably amount of sunlight, and, due to the lattice mismatch between QD and host materials, they often have a detrimental effect on the crystal quality and the device properties. [5, 6] Strain balanced growth has been used before to address this difficulty.[7-9] In this way, we have fabricated solar cells with up to 50 QD layers keeping a reasonable structural quality but still without surpassing the efficiency of a single gap conventional device.[7] Other approaches that may lead to a more favorable strain distribution and better material quality are based on high index and off axis-cut substrates.[10] The best QD-IBSC (efficiency 18.3\%) has been obtained by Blokhin et al.[11]

\section{WHAT IS A QUANTUM POST?}

Quantum posts (QPs) form spontaneously when a very short period InAs/GaAs superlattice (SL) is grown on top of an InAs QDs seed layer (Fig. 1). In some sense, QPS are related with vertically coupled QD (VC-QDs) in the limit of small spacer layer.[12] Due to the strain field generated by the seed, the adatoms of the SL redistribute during growth producing In accumulation on top of QDs and $\mathrm{Ga}$ accumulation around them. As a result, In rich columns are created surrounded by a $\mathrm{Ga}$ rich matrix. The diameter of the columns can be controlled tailoring the size of the QD seeds and the height - the length of the QPs - by varying the number of periods of the SL.

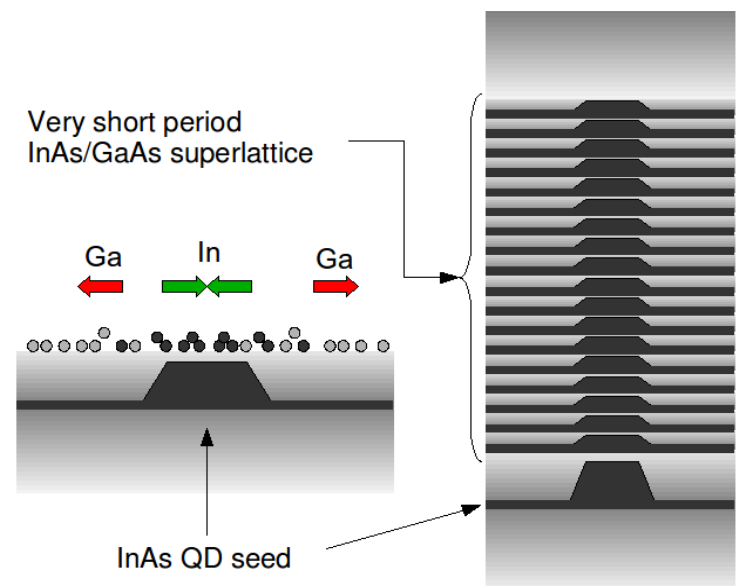

Figure 1 Schema of the quantum post formation.

This technique is very versatile and gives another degree of freedom when growing nanostructures, their height. Normally, dots with small lateral dimension are also very shallow whereas tall QDs have a very large base. Following the QP growth protocol, the diameter of the dot can be varied independently of its height. The former is set by the seed and the latter by the number of periods. For example, it is possible to grow conical nanostructures, dots-in-a-rod or high aspect ratio QDs.[13] 


\section{QUANTUM POSTS IN PHOTOVOLTAICS}

The main interest of using quantum posts instead of quantum dots to form the intermediate band is intimately related with their elongated shape, and hence their potential to tailor the absorption of the photons that cause transitions from the IB to the CB. Typical QDs grown in the Stranski-Krastanov mode have a flat shape with the vertical dimension shorter than the lateral ones. QDs with increased vertical dimension might benefit of a stronger absorption related to IB to CB transitions.[14] The quantum posts presented here are not limited to a precise height and can be a means of producing the desired aspect ratio.

In addition to the above benefit, there is a change in the distribution of the density of states (DOS) of quantum posts. Being elongated structures, they may exhibit 1D behavior for large heights, when the quantum confinement along the post becomes negligible. The limit is roughly imposed by the exciton Bohr radius $(\sim 35 \mathrm{~nm})$, and InAs quantum wires grown on different substrates show clear 1D behavior at relatively short lengths.[15] The advantage of having non-delta-like DOS relies on the larger potential occupation of the ground state instead of only two electrons or holes, which is what can hold the fundamental level in a QD at most. The drawbacks need to be investigated as well since the extra density of states can jeopardize the voltage preservation predicted for the IBSC.

As stated originally by Luque et al [3], in order to keep the open circuit voltage $\left(\mathrm{V}_{\mathrm{oc}}\right)$ of the IBSC only limited by the gap of the host cell ( $\mathrm{GaAs}$ in this case), the IB must be electrically isolated from the $C B$ and the VB. Only radiative transitions between the bands are allowed. In conventional InAs/GaAs QDs, the confinement energies depend strongly on the QD size. For large QDs, the number of energy levels increases and their relative energy spacing diminishes. This favors the carrier thermalization between QD states and continuum states (wetting layer and GaAs barrier) and, as a consequence, degrades the $V_{o c}$ of the device.

For QDs this effect can be avoided by reducing their size and reaching strong quantum confinement regime in the three directions. Small QDs with only one or two confined energy levels and a true OD DOS show experimentally the higher $\mathrm{V}_{\text {oc. }}[11,16,17]$

For similar reasons, the existence of a true 1D DOS in QPs might represent an important drawback for the realization of an IB. In a true 1D DOS there is a continuum of possible electronic states between the bottom of the IB and the $C B$ that allows the mutual exchange of carriers by non radiative transitions. In this case reducing the lateral size of the QPs will reduce the number of 1D energy bands but will not eliminate completely the continuum of states between those bands and the CB.
QPs are good for providing the optimal solution since their height can be tailored independently of their radius. The transition from a OD to a 1D DOS is not an abrupt change and there must be intermediate situations - intermediate lengths for QPs - where the effective density of states increases but without reaching the $E^{-1 / 2}$ dependency of a complete 1D DOS.[18] In this way, an appropriate choice of the QPs length give most of the above mentioned benefits but preserving the $\mathrm{V}_{\mathrm{oc}}$ of the host cell at the same time.

Quantum posts have also another property whose impact in the intermediate band performance needs to be explored. That is their reduced radiative recombination efficiency.

As reported by several authors, due to the strain distribution and the In composition modulation, the ground state electron wavefunction is spread along the whole length of the post whereas the hole wavefunction is confined at the base.[12, 19] If compared with a QD, this charge redistribution decreases the electron-hole overlap and, therefore, the interband matrix element, decreasing the radiative recombination probability in these nanostructures. Accumulation times of several hundreds of microseconds have been found experimentally under moderate positive biases at low temperature.[20]

\section{APROXIMATION TO QPS SOLAR CELLS}

In this section, we present our results on strain balanced quantum posts in the context of photovoltaics. The studied samples do not represent optimized structures, but rather initial attempts In which we focus our attention on the VB to IB transition (recombination, absorption and extraction of carriers) as compared to regular QDs.

\begin{tabular}{|c|c|}
\hline $\begin{array}{c}\text { Window } 26 \mathrm{~nm} \text { p-Ga } \mathbf{0}_{0,51} \mathbf{I n}_{0,49} \mathbf{P} \\
\text { p(Be) } \sim 2 \times 10^{18} \mathrm{~cm}^{-2}\end{array}$ & $\begin{array}{c}\text { Window } 30 \mathrm{~nm} \text { p-Al }{ }_{0,25} \mathbf{G a}_{0,75} \text { As } \\
p(\mathrm{Be}) \sim 1 \times 10^{18} \mathrm{~cm}^{-2}\end{array}$ \\
\hline $\begin{array}{c}\text { Emitter } 100 \mathrm{~nm} \text { p-GaAs } \\
\mathrm{p}(\mathrm{Be}) \sim 4 \times 10^{18} \mathrm{~cm}^{-2}\end{array}$ & $\begin{array}{c}\text { Emitter } 50 \mathrm{~nm} \quad \mathrm{p}-\mathrm{GaAs} \\
\mathrm{p}(\mathrm{Be}) \sim 2 \times 10^{18} \mathrm{~cm}^{-2}\end{array}$ \\
\hline $\begin{array}{c}\text { Intrinsic region } \\
\sim 310 \mathrm{~nm}\end{array}$ & $\begin{array}{l}\text { Intrinsic region } \\
\sim 250 \mathrm{~nm}\end{array}$ \\
\hline $\begin{array}{c}\text { Base } 100 \mathrm{~nm} \mathbf{n} \text {-GaAs } \\
\mathrm{n}(\mathrm{Si}) \sim 1 \times 10^{18} \mathrm{~cm}^{-2}\end{array}$ & $\begin{array}{c}\text { Base } \\
\mathrm{n}(\mathrm{Si}) \sim 5 \times 10^{18} \mathrm{~cm}^{-2}\end{array}$ \\
\hline $\begin{array}{lr}\text { BSF } & 26 \mathrm{~nm} \\
& \mathrm{n}-\mathbf{G a}_{0,51} \mathbf{I n}_{0,49} \mathbf{P} \\
\mathrm{n}(\mathrm{Si}) \sim 2 \times 10^{16} \mathrm{~cm}^{-2}\end{array}$ & 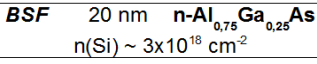 \\
\hline $\begin{array}{c}\text { n-GaAs } \\
\mathrm{n}(\mathrm{Si}) \stackrel{1 \times 10^{18} \mathrm{~cm}^{-2}}{\sim}\end{array}$ & $\begin{array}{c}\text { n-GaAs } \\
\mathrm{n}(\mathrm{Si}) \stackrel{1 \times 10^{18} \mathrm{~cm}^{-2}}{\sim}\end{array}$ \\
\hline
\end{tabular}

Figure 2 Structure of the devices. Samples A, B and D follow the structure on the left and sample $C$ the one on the right. 


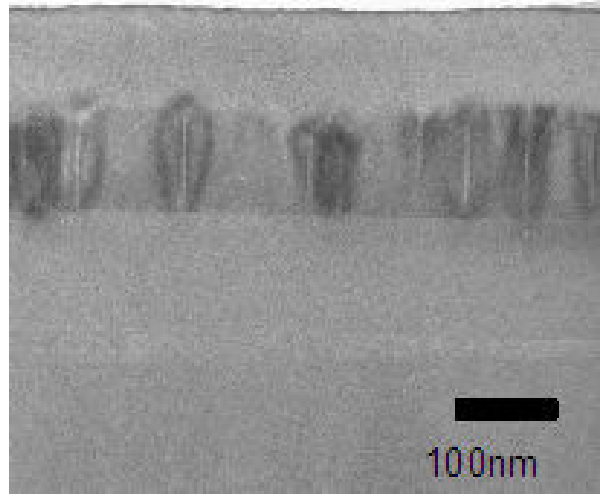

Figure 3 Bright field TEM image of several QPs

\section{Samples growth}

The samples were grown by solid source molecular beam epitaxy (MBE) on Si doped GaAs (001) substrates. All samples were grown using strain balance techniques to avoid the detrimental effect of stacking multiple strained layers as explained above.[5,7,8] The structure, composition and doping level of the samples can be seen in figure 2. The nanostructures were grown in the intrinsic region of the solar cell according to the following sequences. The QDs sample (sample A) was grown starting with $4 \mathrm{~nm}$ of GaAs followed by $2 \mathrm{ML}$ of InAs. After formation of the QDs, they were capped by $8 \mathrm{~nm}$ of GaAs and $3 \mathrm{~nm}$ of $\mathrm{GaAs}_{1-\mathrm{x}} \mathrm{P}_{\mathrm{x}}(\mathrm{x} \sim 18 \%)$ as strain compensating layer. This sequence leads to a spacer thickness of $15 \mathrm{~nm}$ between QD layers which we repeated 17 times. For the QPs (samples B and C), the growth sequence starts with 2 $\mathrm{ML}$ of InAs to grow the QDs seed layer. After formation of the seed, we deposited a very short period superlattice of $0,85 \mathrm{~nm}$ of $\mathrm{GaAs}_{1-\mathrm{x}} \mathrm{P}_{\mathrm{x}}(\mathrm{x} \sim 14 \%)$ and $0.22 \mathrm{~nm}$ of InAs completing 175 periods for sample $B$ and 100 periods for sample C. For all samples, GaAs intrinsic regions were introduced to separate the nanostructures from the base and the emitter. This GaAs barrier was $25-\mathrm{nm}$-thick below and above in samples $A$ and $B$, whereas sample $C$ has $a$ barrier of $110 \mathrm{~nm}$ below and $20 \mathrm{~nm}$ above the nanostructures. We grew also a reference $\mathrm{GaAs}$ cell with the same geometry that samples A and B (sample D) but only with $\mathrm{GaAs}$ in the intrinsic region. The substrate temperature was kept at $510^{\circ} \mathrm{C}$ during the growth of the nanostructures and the spacers and at $600^{\circ} \mathrm{C}$ for the rest of the structure. The $\mathrm{As}_{4}$ beam equivalent pressure was $1.5 \times 10^{-6}$ mbar in all cases.

As it can be seen in Figure 2, the p-i-n diodes are very thin $(<500 \mathrm{~nm})$ so changes in the intrinsic region absorption or emission properties should have a large impact on the device characteristics. The use of phosphorus in the spacer increases the overall crystal quality and allows the growth of longer quantum posts. The degree of strain compensation in the three samples is $\sim 60 \%$ as revealed by in-situ accumulated stress measurements (not shown).

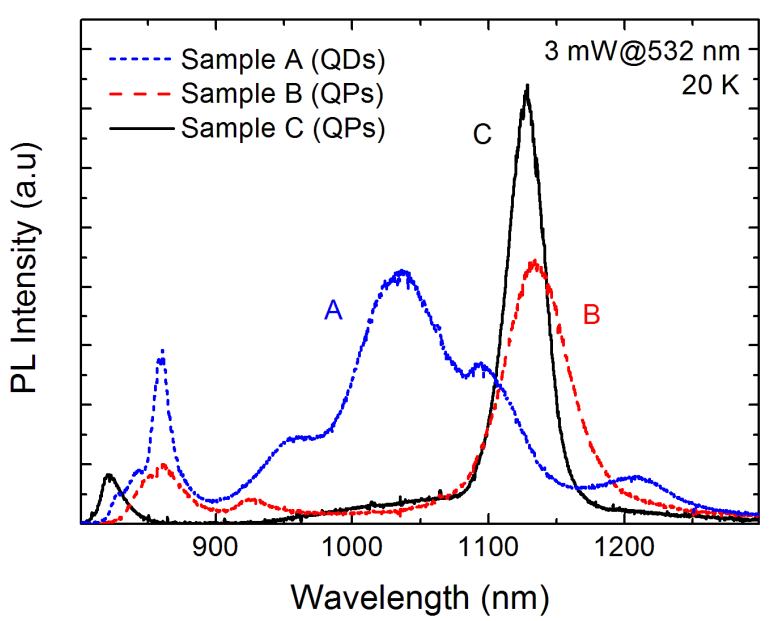

Figure 4 Comparison of the low temperature photoluminescence

\section{Structural and optical properties}

Figure 3 shows a transmission electron microscope (TEM) image of sample $\mathrm{C}$. Bright columns are clearly visible across the intrinsic region indicating the successful formation of extremely long QPs (around $120 \mathrm{~nm}$ ). The region between the posts forms a wide InGaAsP quantum well consequence of the mixing of the remaining In and the GaAsP of the barriers in the SL. The strain field dominates the image contrast around each QP introducing uncertainty in the nanostructure diameter which we estimate $\sim 20 \mathrm{~nm}$.

Very few dislocations are visible in the upper part of the device $\left(\sim 10^{7} \mathrm{~cm}^{-2}\right)$ indicating good crystal quality for QPs of this length. Nevertheless, keeping constant the degree of strain compensation, we observe that the defect density increases with the number of periods, as expected.

The photoluminescence (PL) spectra excited with a 532 $\mathrm{nm} \mathrm{CW}$ laser at $20 \mathrm{~K}$ are shown in Figure 4 for the different samples. PL of sample A suggests the existence of QDs families of different size, probably corresponding to different QD layers. This evolution of the QD size with the number of layers is often found in closely spaced stacks of QDs and is a consequence of In segregation and the strain field of the buried dots.[19] Both QPs samples, on the contrary, show a narrower emission band centered at $1130 \mathrm{~nm}$ (with a full width at half maximum of $33 \mathrm{~nm}$ for sample $\mathrm{C}$ ) which is consistent with strong electronic coupling between the layers. The peak corresponding to sample $B$ is slightly broader than that of sample $C$, but, the integrated PL is the same in both QPs samples and approximately half of the integrated emission of the QDs families in sample $A$. 


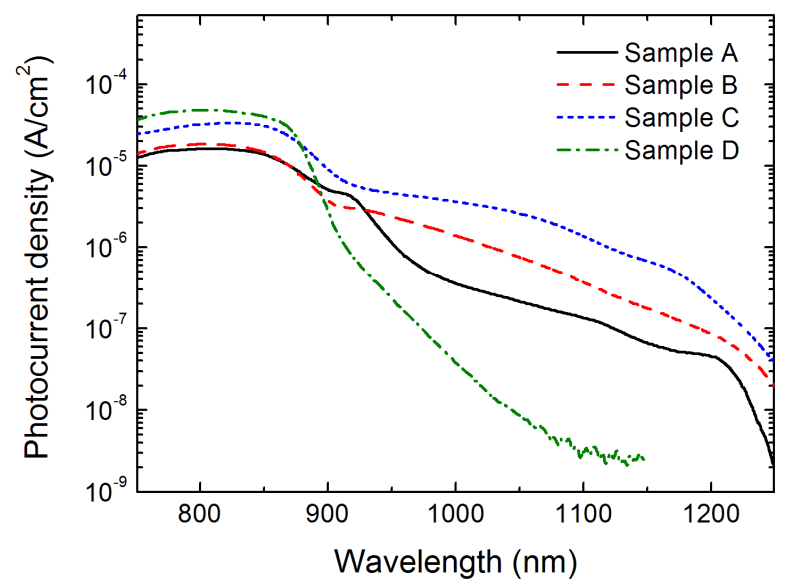

Figure 5 Comparison of the room temperature photocurrent.

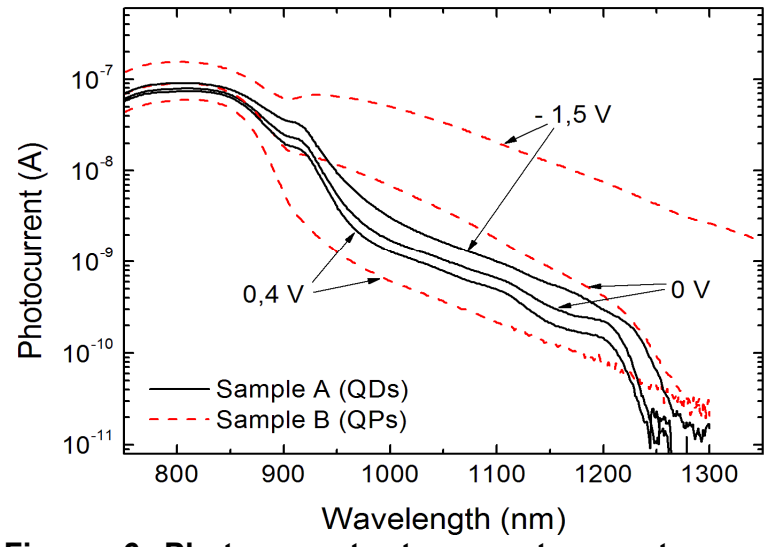

Figure 6 Photocurrent at room temperature as a function of the applied bias

\section{Photocurrent experiments}

Photocurrent (PC) measurements give information not only of the absorption spectrum of the samples but also of the extraction mechanisms that govern carrier transport from the nanostructures to the device contacts. To this aim, 700 micron wide diodes (400 micron for sample C) were fabricated by standard UV lithography, wet etching and electron beam metal deposition. The optical window was 600 micron in diameter (300 micron). There was no antireflecting coating or metal grid to reduce the series resistance of the devices.

For an ideal IBSC, there should not be photocurrent at all below the bandgap except in the case of two photon absorption. In practice, however, all IBSCs show some single photon thermally and/or tunneling assisted photocurrent below the gap which, in turn, serves as a diagnosis of the performance of the nanostructures as light absorbers.[21]
Figure 5 shows the room temperature photocurrent density spectrum in logarithmic scale of the three samples considered. The GaAs reference sample is included for comparison. Due to the short length of the emitter in our samples, the above gap efficiency depends crucially on the composition of the intrinsic region. Above the GaAs edge, the photocurrent of samples $A$ and $B$ show a factor three reduction when compared with the reference GaAs cell (sample D). This might be attributed to carrier trapping and point defects introduced during the growth of the nanostructures. [7] The reduction is less drastic in sample $\mathrm{C}$, although it can not be compared directly with the other samples due to its different design (see Figure 2). Nevertheless, the most relevant information is hindered below the gap. All the samples show intense absorption down to $1200 \mathrm{~nm}$ largely exceeding the GaAs reference solar cell. In this range samples $A$ to $C$ are comparable since the intrinsic region has similar. There are important differences among them, though. Both QPs samples show more photocurrent than the QD sample in the whole range being up to one order of magnitude in the case of sample C.

The processes that govern the extraction of carriers can be further investigated measuring the $P C$ as a function of the applied voltage in samples $A$ and $B$. In Figure 6, the PC of the QPs sample is greatly enhanced applying negative bias and can reach an important fraction of the current at the GaAs band edge. Due to the large electrical polarizability of QPs along the growth direction, absorbed electron hole pairs and carriers trapped by the QP from the surrounding regions are readily separated before recombination occurs. This charge separation effect, absent in shallow QDs, might be interesting to facilitate minority carrier extraction, minimizing the recombination probability in the nanostructure or the trapping by defects in the matrix. Tunneling, however, must be avoided to prevent the degradation of the $\mathrm{V}_{\mathrm{oc}}$. The usage of a field damping layer at one end of the intrinsic region or a higher bandgap material in the barrier may serve this purpose.[22]

The evolution of QDs PC with the applied bias is much less pronounced, as shown in Figure 6. The weak dependence with voltage might indicate that the extraction is mainly due to thermally assisted escape.

To assess the role of thermally assisted processes in both kind of nanostructures, we show the temperature evolution of the photocurrent of sample A and B in Figures 7(a) and (b), respectively. For the QDs the signal increases in the whole wavelength range as the temperature rises indicating that the extraction of carriers from the nanostructures is a combination of thermal and tunnel assisted photocurrent.[21] On the contrary, the extraction of carriers from the QPs is almost constant at all temperatures, increasing slightly only for the longer wavelengths. In this case the predominant extraction mechanism is tunnel across the GaAs barriers, having the thermal extraction a minor importance. 
We tentatively conclude from these experiments that unwanted photocurrent from the IB in QPs solar cells is largely dominated by the electric field. To prevent the extraction of carriers below the bandgap and the resulting drop in $V_{\text {oc }}$ in a QPs solar cell, it might be enough to increase the barrier height or to introduce a field damping layer above the QPs layer. This implies a modification of the solar cell design and not of the nanostructures. In QDs, where the dominant mechanism at room temperature is thermal extraction, preventing the drop in $V_{\text {oc }}$ requires almost exclusively a change in the nanostructures themselves with the associated modification of their spectral response.[16, 17, 21] Additional time-resolved photoluminescence characterization of our samples should confirm the conclusions derived here.
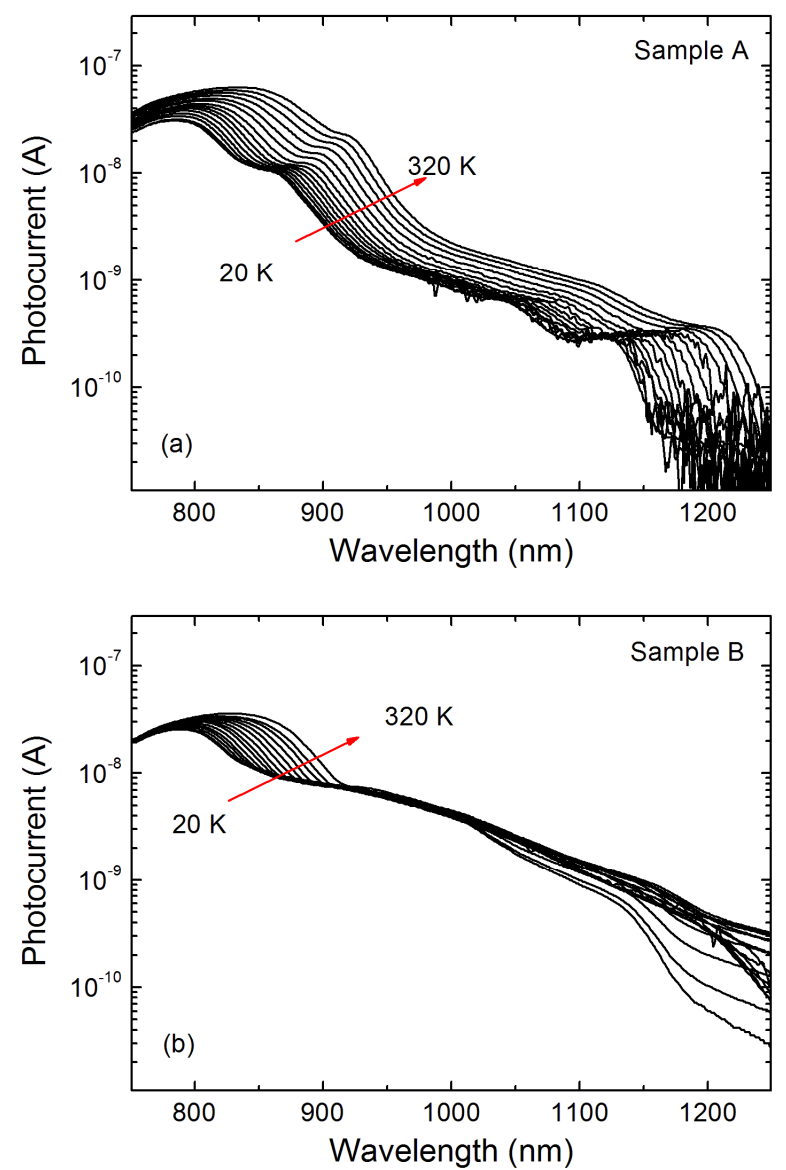

Figure 7 Temperature dependence of the photocurrent for (a) sample A and (b) sample B.

\section{CONCLUSIONS}

In this work we have presented our initial studies on solar cells containing strain balanced InAs quantum posts. After introducing the physical concepts that makes them of interest for the realization of the intermediate band solar cell, we present practical examples of QPs devices that exhibit high photocurrent below the bandgap. The main drawback of the IBSC approach followed here is the drop in the $V_{o c}$ consequence of the tunneling of carriers out of the nanostructures before the second photon absorption occurs. We propose the use of field damping layers or high energy bandgap barrier materials to overcome these difficulties

\section{ACKNOWLEDGEMENT}

We acknowledge support by MICINN (NANOGEFFES ENE2009-14481-C02-02, TEC2008-06756-C03-02/TEC, Consolider GENESIS MEC CSD2006-0004, Consolider CSD2009-00013), CAM (Numancia 2 S2009/ENE-1477), CSIC (PIF 200950I154) and Junta de Andalucía (PAI research group TEP-120, project P08-TEP-03516).

\section{REFERENCES}

[1] B. Browne, A. loannides, J. Connolly, K. Barnham, J. Roberts, R. Airey, G. Hill, G. Smekens, and J. Van Begin, "Tandem quantum well solar cells", 33rd IEEE Photovoltaic Specialists Conference, pp 11-16 (2008).

[2] A. Marti, L. Cuadra, and A. Luque, "Quantum dot intermediate band solar cell", 28th IEEE Photovoltaic Specialists Conference, pp 940-943 (2000).

[3] A. Luque and A. Martí, "Increasing the efficiency of ideal solar cells by photon induced transitions at intermediate levels", Phys. Rev. Lett, 78, 5014-5017 (1997).

[4] A. Luque, P. G. Linares, E. Antolín, E. Cánovas, C. D. Farmer, C. R. Stanley and A. Martí, Appl. Phys. Lett. 96, 013501 (2010).

[5] J. T. Ng, U. Bangert, and M. Missous, "Formation and role of defects in stacked large binary InAs/GaAs quantum dot structures", Semiconductor Science and Technology, 22, pp. 80-85 (2007).

[6] A. Martí, N. López, E. Antolín, E. Cánovas, A. Luque, C. R. Stanley, C. D. Farmer, and P. Díaz, "Emitter degradation in quantum dot intermediate band solar cells," Appl. Phys. Lett., 90, 233510 (2007).

[7] D. Alonso-Álvarez, A. G. Taboada, J. M. Ripalda, B. Alén, Y. González, L. González, J. M. García, F. Briones, A. Martí, A. Luque, A. M. Sánchez, and S. I. Molina. "Carrier recombination effects in strain compensated quantum dot stacks embedded in solar cells", Appl. Phys. Lett. 93, 123114 (2008).

[8] R. Oshima, A. Takata, and Y. Okada, "Straincompensated InAs/GaNAs quantum dots for use in highefficiency solar cells", Appl. Phys. Lett. 93, 083111 (2008).

[9] S. M. Hubbard, C. D. Cress, C. G. Bailey, R. P. Raffaelle, S. G. Bailey, and D. M. Wilt, "Effect of strain 
compensation on quantum dot enhanced GaAs solar cells," Appl. Phys. Lett., 92, 123512, (2008).

[10] V. Popescu, G. Bester, M. C. Hanna, A. G. Norman, and A. Zunger, "Theoretical and experimental examination of the intermediate-band concept for strain-balanced (In, Ga)As/Ga(As,P) quantum dot solar cells", Phys. Rev. B 78, 205321 (2008).

[11] S. A. Blokhin, A. V. Sakharov, A. S. P. A. M. Nadtochy, M. V. Maximov, N. N. Ledentsov, A. R. Kovsh, S. S. Mikhrin, and V. M. Lantrat, "AIGaAs/GaAs Photovoltaic Cells with an Array of InGaAs QDs," Semiconductors, 43, pp. 514-518 (2009).

[12] L. H. Li, G. Patriarche, N. Chauvin, P. Ridha, M. Rossetti, J. Andrzejewski, G. Sek, J. Misiewicz,, and A. Fiore, "Controlling the Aspect Ratio of Quantum Dots: From Columnar Dots to Quantum Rods", IEEE Journal of Selected Topics in Quantum Electronics, 14, pp 12041213 (2008)

[13] L. H. Li, P. Ridha, G. Patriarche, N. Chauvin, and A. Fiore, "Shape-engineered epitaxial InGaAs quantum rods for laser applications", Appl. Phys. Lett, 92, 112102 (2008).

[14] A.Luque, A. Martí, E. Antolín and P. García-Linares, "Intraband Absorption for Normal Illumination in Quantum Dot Intermediate Band Solar Cells," Solar Energy MAterials and Solar Cells (To be published) (2010).

[15] B. Alén, D. Fuster, G. Muñoz-Matutano, J. MartínezPastor, Y. González, J. Canet-Ferrer, and L. González , "Exciton Gas Compression and Metallic Condensation in a Single Semiconductor Quantum Wire" Phys. Rev. Lett., 101, 067405 (2008).

[16] S.M. Hubbard, C.G. Bailey, R. Aguinaldo, S. Polly, D. V. Forbes, and R. P. Raffaelle, "Characterization of quantum dot enhanced solar cells for concentrator photovoltaics", $34^{\text {th }}$ IEEE Photovoltaic Specialists Conference, 7-12 (2009).
[17] D. Guimard, R. Morihara, D. Bordel, K. Tanabe, Y. Wakayama, M. Nishioka, and Y. Arakawa, "Fabrication of InAs/GaAs quantum dot solar cells with enhanced photocurrent and without degradation of open circuit voltage" Appl. Phys. Lett. 96, 203507 (2010).

[18] S. J. Lee, N. H. Shin, J. J. Kot, M. J. Parkt and R. Kiimmel, "Density of states of quantum dots and crossover from 3D to QOD electron gas" Semicond. Sci. Technol. 7 1072-1079 (1992).

[19] J. He, H. J. Krenner, C. Pryor, J. P. Zhang, Y. Wu, D. G. Allen, C. M. Morris, M. S. Sherwin, and P. M. Petroff, "Growth, structural, and optical properties of selfassembled (In, Ga)As quantum posts on GaAs", Nanoletters, 7(3), 802-806 (2007).

[20] H. J. Krenner, C. E. Pryor, J. He, and P. M. Petroff, "A Semiconductor Exciton Memory Cell Based on a Single Quantum Nanostructure" Nanoletters, 8(6) 1750-1755 (2008).

[21] E. Antolín, A. Martí, C. D. Farmer, P. G. Linares, E. Hernández, A. M. Sánchez, T. Ben, S. I. Molina, C. R. Stanley, and A. Luque, "Reducing carrier escape in the InAs/GaAs quantum dot intermediate band solar cell," $J$. Appl. Phys., (to be published) (2010).

[22] A. Martí, E. Antolín, E. Cánovas, N. López, P. G. Linares, A. Luque, C. R. Stanley, and C. D. Farmer, "Elements of the design and analysis of quantum-dot intermediate band solar cells," Thin Solid Films, 516, 20 (2008). 